\title{
El emprendimiento tecnológico en Suramérica: una aproximación a sus determinantes individuales
}

\section{Guillermo Zapata, ${ }^{*}$ Sara Fernández López, ${ }^{* *}$ Isabel Neira Gómez***}

Perfiles Latinoamericanos, 26(52) | 2018

DOI: $10.18504 / \mathrm{pl} 2652-003-2018$

Recibido: 9 de junio de 2015

Aceptado: 16 de enero de 2018

\begin{abstract}
Resumen
Este artículo analiza los determinantes individuales del emprendimiento tecnológico en Suramérica y si difieren de aquellos que impulsan el emprendimiento convencional. Con esto se contribuye a la comprensión de las nuevas empresas de base tecnológica (NEBT) en pro de su impulso para fomentar en la región economías que se basen en la innovación. Utilizando modelos de regresión logística y los datos del Global Entrepreneurship Monitor (GEM) para 2011, se ha encontrado que la situación laboral, la renta, las habilidades y el conocimiento determinan el emprendimiento de nuevos negocios, y que el género, el nivel de estudios y la edad del emprendedor determinan que un negocio sea de base tecnológica.
\end{abstract}

\begin{abstract}
This paper analyses the individual determinants of technological entrepreneurship and whether they differ from those of the conventional entrepreneurship in South America. This paper contributes to the understanding of New Technology-Based Firms (NEBTs) to foster innovationbased economies in the region. Using logit models and the data from Global Entrepreneurship Monitor (GEM) 2011, we find that employment status, income, perceived skills and meeting an entrepreneur are driving forces for entrepreneurship. However the gender, age and studies exclusively impact on technological entrepreneurship.
\end{abstract}

Palabras clave: emprendimiento, emprendedores, empresa de base tecnológica, investigación y desarrollo, datos estadísticos, innovaciones tecnológicas, América del Sur.

Keywords: Entrepreneurship, entrepreneurs, new technological based firm, statistical data, technological innovations, South America.

* Doctor en Economía por la Universidad de Santiago de Compostela. Departamento de Economía Financiera y Contabilidad, Universidad de Santiago de Compostela | guillermo.zapata@usc.es

** Doctora en Economía por la Universidad de Santiago de Compostela. Departamento de Economía Financiera y Contabilidad, Universidad de Santiago de Compostela | sara.fernandez.lopez@usc.es

*** Doctora en Economía por la Universidad de Santiago de Compostela. Departamento de Economía Cuantitativa, Universidad de Santiago de Compostela | sara.fernandez.lopez@usc.es 


\section{Introducción}

E

interés del presente artículo parte de sucesos económicos acaecidos en las últimas décadas que han contribuido al desarrollo económico y se han atribuido a las empresas de reciente creación (Giovannetti et al., 2011; Rueda et al., 2014), en especial a las de contenido tecnológico o de base tecnológica (Aspelund et al., 2005; Bower, 2003; Storey \& Tether, 1998). Tales beneficios se reflejan como apertura de nuevos mercados, competitividad económica (Cooper, 1971; Autio, 1997), empleos de calidad, cambio estructural y como fuente de innovación y cambio tecnológico (Gassler, 1998), todo enmarcado en el nuevo modelo productivo cuya base es el conocimiento, la investigación y la innovación, elementos vitales para el bienestar y competitividad de la economía moderna (OCDE, 2009).

Así, aquí se aborda la identificación de los factores individuales que determinan la creación de nuevas empresas de base tecnológica (NEBT) en Suramérica, y se analiza en qué magnitud esos factores que son propios del emprendimiento tecnológico se diferencian de los que impulsan un emprendimiento convencional. La finalidad es aproximarnos al conocimiento del perfil del emprendedor tecnológico suramericano.

Existen estudios acerca de dicho tema, aunque son sobre todo de carácter descriptivo (Westheady, 1994; Harvey, 1994; Rodriguez et al., 2013; Zapata et al., 2014a; Donckels, 1989; Autio et al., 1989; Ortín et al., 2008; Goldin, 2006; Fagenson \& Jackson, 1993) y de contextos de economías desarrolladas como la Unión Europea y Estados Unidos (Ferreira et al., 2014). La propuesta de este documento es distinta en tanto que se trata de un análisis empírico de una muestra representativa de la población adulta suramericana (Argentina, Brasil, Chile, Colombia, Perú, Uruguay y Venezuela) en el que se usan datos del Global Entrepreneurship Monitor (GEM) para el año 2011. Con ese fin se utiliza aquí un modelo de elección discreta, mismo que permite identificar cuál es la probabilidad de ser emprendedor cuando se cumple una serie de criterios. La aportación (inédita en la literatura) consiste en señalar el diferencial entre los emprendedores tradicionales y los que apuestan por empresas tecnológicas.

Luego de esta introducción, el artículo prosigue con una revisión de la literatura más relevante acerca de los principales factores individuales que determinan el emprendimiento tecnológico y de dónde han surgido las variables objeto del presente estudio. En una sección posterior se describen los datos utilizados y la muestra, además de que se definen las variables explicadas y explicativas. Enseguida se exponen las consideraciones metodológicas empleadas en el análisis econométrico y se describen los resultados obtenidos, lo que da lugar a su 
análisis y discusión. Finalmente, en las conclusiones se resumen las aportaciones y recomendaciones para la futura investigación del tema.

\section{Revisión de la literatura}

Es conocido el crecimiento económico de Suramérica de los últimos años (OECD/ECLAC, 2012; CEPAL, 2014) y que la economía de esta región en su mayor parte se cimienta en pequeńos negocios, lo que caracteriza a su población como emprendedora (Lederman et al., 2014). Esto se corresponde con lo que dice la literatura, la cual ha resaltado la importancia de la nueva empresa por su contribución al empleo y crecimiento económico (Alonso et al., 2006; Costa, 2006). Sin embargo, actualmente los países líderes vienen siguiendo un nuevo modelo productivo que se fundamenta en el conocimiento y la innovación, con soporte en la investigación científica que, al vincularla al desarrollo tecnológico y a la empresa, logra ser fuente de desarrollo económico. Así lo planteó Vannervar en 1945 en el informe Science: The Endless Frontier (Ciencia: la interminable frontera). En este proceso, las empresas innovadoras de nueva creación juegan un papel de primer orden, en especial las de base tecnológica (Aspelund et al., 2005; Bower, 2003; Storey y Tether, 1998), donde el conocimiento, sobre todo el económicamente útil y sus externalidades, cumple un rol vital en el crecimiento y en el desarrollo económico y social de una región (Hülsbeck \& Kitzinger, 2011). Es por ello que se suele atribuir mayor potencial a las NEBT por encima de otras nuevas en general (Oakey, 1995); así mismo, Almus \& Nerlinger (1999) resaltan la importancia de ese tipo de empresas para la economía, pues las considera primera fuente de nuevo empleo y motor de cambio tecnológico y crecimiento económico.

En ese sentido, se han publicado estudios del emprendimiento tecnológico o creación de NEBT, por ejemplo, los de Ferreira et al. (2014) y Cunha et al. (2013); sin embargo, todavía son escasos en comparación con los del emprendimiento en general. Y en cuanto a los factores individuales del emprendimiento tecnológico (ET), adoptan un enfoque descriptivo (Westhead \& Storey, 1994; Harvey, 1994; Rodríguez et al., 2013; Zapata et al., 2014a; Donckels, 1989; Autio et al., 1989; Ortín et al., 2008; Goldin, 2006; Fagenson \& Jackson, 1993) y no se conoce alguno dedicado a Suramérica. Solo Zapata et al. (2014b) elaboran un análisis econométrico de los factores individuales determinantes del ET para el caso español, en donde hallan factores recurrentes.

El objetivo de este artículo es entonces analizar los factores determinantes del emprendimiento tecnológico. En relación con ello, la literatura ha señalado que uno de los factores individuales que más contribuye al desarrollo de iniciativas 
emprendedoras es la educación (Rueda et al., 2014). Esto es, que la creación de NEBT requiere de conocimientos técnico-científicos que, en principio, tienen que aportar sus fundadores, los cuales, en consecuencia, deben poseer elevados niveles de educación. Esto lo han evidenciado Storey \& Tether (1998) en su revisión de las NEBT de Europa, cuando muestran que los emprendedores de este tipo de iniciativas presentan ese rasgo educativo. A su vez, Fagenson $\&$ Jackson (1993) y Goldin (2006) han encontrado que es el nivel de educación lo que ha aumentado de manera espectacular la participación de las mujeres como propietarias de nuevos negocios en sectores de alta tecnología. En tanto que Storey \& Tether (1998) han dejado en claro que los emprendedores de las NEBT muestran niveles de educación más elevados en comparación con los de otro tipo de negocios; y Westhead \& Storey (1994), en su investigación para el Reino Unido, indican que el $48 \%$ de los fundadores de NEBT tenían doctorado. Ortín et al. (2008), por su parte, destacan la presencia de un elevado número de doctores en la creación de spin-offs de base tecnológica para el caso español, y Zapata et al. (2014b) lo hacen para las NEBT.

Un papel semejante lo tienen la edady la experiencia laboral de los emprendedores. Así, la edad media de los individuos involucrados en la creación de NEBT suele situarse entre los 30 y los 50 años (Westhead \& Storey, 1994; Harvey, 1994; Donckels, 1989; Autio et al., 1989; Ortín et al., 2008; Zapata et al., 2014b), lo que coincide de cierto modo con los emprendedores de otro tipo de negocios que tienden a ser personas jóvenes. Sin embargo, como destacan Storey \& Tether (1998), es poco probable encontrar emprendedores muy jóvenes (con edades menores a 25 años), cuya presencia en otro tipo de negocios oscila en torno a un $15 \%$. Dado que el perfil del emprendedor de empresas de NEBT requiere de un determinado nivel educativo, suele suceder que ello solo se alcanza cuando se han superado los 25 años. Es más, cuando las NEBT hacen referencia a spin-offs universitarias, la mayor edad vendría también explicada porque el emprendedor ha dado más tiempo de servicio en la universidad (Aceytuno \& Paz, 2008). Esto se encuentra estrechamente ligado a la experiencia previa, por eso Colombo \& Grilli (2005) señalan que lo que saben y pueden hacer los fundadores de las nuevas empresas se relaciona bastante con lo que aprendieron de su antiguo trabajo en alguna organización, y de esto son evidencia los spin-offs de base tecnológica (Ortín et al., 2008; Clarysse \& Moray, 2004). Ello tal vez se debe a que dichas empresas desempeñan su actividad en un entorno muy competitivo donde estas habilidades gestoras y tecnológicas son fundamentales (Gompers et al., 2005).

El emprendimiento se asocia a la iniciativa y el optimismo que, por lo general, surgen del bagaje de habilidades personales que el emprendedor potencial cree poseer (Álvarez \& Urbano, 2011; Rueda et al., 2014). En ese sentido, 
estudios como el de Almus \& Nerlinger (1999) plantean como hipótesis que son las habilidades técnicas y de ingeniería las que influyen en el crecimiento de las NEBT. En una industria tan incierta como la alta tecnología, la existencia de las oportunidades emprendedoras responde a que hay quienes creen tener conocimientos y habilidades que otros carecen y que podrían crear valor si se combinan con otros recursos (Shane \& Venkataraman, 2000; Alvarez \& Barney, 2002).

Los individuos con pocos recursos económicos tendrán más dificultades para crear sus negocios (Ortín et al., 2007) en comparación con aquellos con mayor riqueza (renta); estos últimos podrían financiar las operaciones de una eventual empresa (Colombo \& Grilli, 2005) y tendrían menos restricciones dado que su capital representaría un colateral. Y puesto que las NEBT conllevan un fuerte riesgo asociado a su puesta en marcha y una gran incertidumbre en cuanto a sus resultados (Ortín et al., 2008), cabe esperar que su acceso al crédito sea muy limitado, lo que agrava la situación de quienes cuentan con pocos recursos financieros.

En cuanto al género, existe un mayor porcentaje de iniciativas emprendedoras masculinas (Westhead \& Storey, 1994; Harvey, 1994; Rodríguez et al., 2013; Zapata et al., 2014b). Según Rodríguez et al. (2013), la menor presencia femenina se debe a las barreras tradicionalmente asociadas a las mujeres que, cuando se trata del emprendimiento tecnológico, se agudizan. Así al sector de alta tecnología se le atribuye: $a$ ) una naturaleza más competitiva con dificultad para emprender (Mayer, 2008; Zhao et al., 2005); b) conflictos entre la rigidez de la dedicación y las cargas familiares (Mayer, 2008), y c) altas exigencias de inversión por el alto riesgo asociado (Ruiz et al., 2012), debido a que se les atribuyen escasa o nula experiencia, en sectores competitivos (Neergaard et al., 2006). Entre otras razones específicas también se encuentra una reducida presencia en titulaciones técnicas, de donde emerge mayoritariamente este tipo de emprendimientos (Greene, 2000), y una menor tasa de presentación de tesis doctorales que es una fuente de concepción de NEBT (Vaquero et al., 2011). De igual modo, los roles tradicionales conferidos a las mujeres han obstaculizado la comercialización de sus investigaciones (Murray \& Grahan, 2007).

Conocer personalmente a un emprendedor es un factor que fortalece las habilidades, hábitos y actitudes de cara a poner en marcha una empresa (Gibb, 1997; Álvarez \& Urbano, 2011), mientras que tener antecedentes familiares forma parte del bagaje emprendedor y se explica por el aprendizaje social (Bandura, 1977). Roberts (1991) señala que esto puede ser determinante para fundar una NEBT; sin embargo, este factor netamente social va más allá, puesto que forma parte del capital social y de las redes sociales. La participación en estas asimismo es un aspecto crítico en la decisión de convertirse en empresario y en el resultado posterior (Aldrich, 2012; Davidsson \& Honig, 2003; Lechner et al., 2006; Jack \& Anderson, 2002; Aldrich \& Fiol, 1994; Aldrich \& Zimmer, 1986). 
Ahora bien, en este artículo se considera como variable de control el territorio. A pesar de no ser el objetivo específico de este trabajo, hay una creciente literatura acerca del clustering (agrupamiento) espacial de la innovación centrada en explicar las ventajas regionales por las externalidades positivas de localización o la retroalimentación generada por algún shock (suceso) inicial. Xue \& Klein (2010) dan cuenta de que en determinados sectores tecnológicos hay una relación positiva entre la disponibilidad de los recursos estratégicos, medidos mediante la inversión regional en I+D, y la actividad emprendedora de base tecnológica (Petti \& Zhang, 2011; Venkataraman, 2004). Así lo refieren también, aunque para los spin-offs, Goldfarb \& Henrekson (2003) y O'Shea et al. (2008), autores que resaltan la importancia de factores intrínsecos de la región como determinantes de su capacidad emprendedora.

Esta revisión previa de la literatura, a diferencia de otras investigaciones, ha puesto el énfasis en la justificación de por qué los factores individuales más recurrentes en los estudios de la creación de empresas tendrían un efecto incluso mayor cuando se trata de NEBT. De confirmarse estas hipótesis, se estarían perfilando los rasgos que identifican al potencial emprendedor tecnológico y en qué medida este se diferencia del emprendedor convencional.

\section{Datos y estadísticos descriptivos}

\section{La base de datos y la muestra}

La base de datos que se utiliza en este análisis fue desarrollada por el Global Entrepreneurship Monitor (GEM), proyecto que evalúa anualmente la actividad emprendedora de individuos en todo el mundo. Son datos que se recogen mediante la Encuesta de Población Adulta (APs, por su sigla en inglés). En el caso presente, la información procede de una muestra representativa de 27653 individuos residentes en Suramérica (Argentina, Brasil, Chile, Colombia, Perú, Uruguay y Venezuela), con edades, en 2011, de entre 18 y 64 años. De forma complementaria, para los datos regionales se ha utilizado información de la Red de Indicadores de Ciencia y Tecnología-Iberoamericana e Interamericana (RICYT) correspondiente a 2011, y del informe Índice de Innovación Global (GII, por su sigla en inglés) en su edición 2012.

Se considera que la información del GEM es adecuada para este trabajo porque se ajusta a los perfiles elegidos: es el máximo estudio del emprendimiento a nivel mundial que mide la actividad empresarial, aspiraciones y actitudes de individuos en diversos países, y permite realizar análisis regionales y compara- 
tivos dada la homogeneización de los datos y el uso de una misma metodología para todos los países participantes.

\section{Variables consideradas y análisis descriptivo}

El proyecto GEM busca determinar la intencionalidad emprendedora de la población adulta de un territorio mediante el índice de la actividad emprendedora incipiente o, en su terminología en inglés, Total Early-Stage Entrepreneurial Activity (TEA). Por tanto, nuestra variable dependiente (TEA11) mide el emprendimiento de la población adulta por medio de una variable dicotómica que diferencia entre los emprendedores (1) y los no emprendedores (0), en función de si el individuo tiene o no participación en actividades emprendedoras tempranas (hasta los 42 meses).

Dentro del índice TEA puede identificarse el nivel tecnológico de la iniciativa emprendedora a través del sector en el que se ubica (TEA11TEC), seleccionando entre las respuestas "Sector no tecnológico o de baja tecnología” y "Sector de media o alta tecnología”, de acuerdo a la clasificación propuesta por la OCDE. De esta forma, con la variable TEA11TEC aquí se ha dividido la muestra en dos. La primera submuestra diferencia a los emprendedores tecnológicos, esto es, a aquellos cuyas iniciativas pertenecen a un "Sector de alta o media tecnología", y a los no emprendedores. La segunda, diferencia a los emprendedores "no tecnológicos", es decir, a aquellos cuyas iniciativas pertenecen a un "Sector no tecnológico o de baja tecnología”, y a los no emprendedores.

Gráfica 1. Nivel tecnológico del emprendimiento en Suramérica por países (\%)

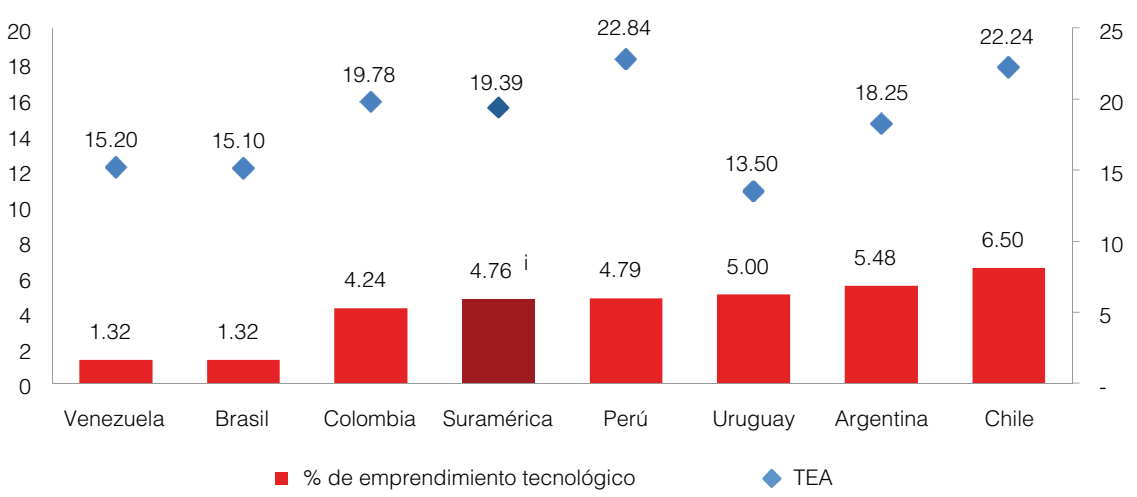

'Este resultado hay que interpretarlo con cautela debido a que ha sido calculado mediante una media aritmética con base en las todas las observaciones individuales de los países participantes, donde Colombia y Chile, por sus estudios regionales, cuentan con una muestra mayor en comparación con los demás países.

Fuente: Elaboración propia.

G. Zapata, S. Fernández López, I. Neira Gómez | El emprendimiento tecnológico en Suramérica: una aproximación a sus determinantes individualesa | Perfiles Latinoamericanos, 26(52) | FLACso México 
En 2011, el índice TEA (TEA11) alcanzó para Suramérica un valor de $19.39 \%$ de toda la población. En tanto que el TEA correspondiente a las iniciativas ubicadas con niveles de media y alta tecnología (TEA11TEC) representó el $0.92 \%$. Por tanto, del total de iniciativas emprendedoras iniciadas en 2011, $4.76 \%$ sería de base tecnológica.

Las variables independientes utilizadas correspondientes a los factores detectados en el marco teórico se distribuyen en dos grupos: las individuales y las regionales (variables de control). Ambas se resumen en el cuadro 1.

Cuadro 1. Definición de las variables independientes utilizadas en el análisis

\begin{tabular}{|c|c|c|c|}
\hline Tipo de factor & Variable & Descripción de variable & Categorías \\
\hline \multirow{21}{*}{ Individuales } & \multirow{2}{*}{ Género } & \multirow{2}{*}{ ¿Cuál es su género? } & Hombre (0) \\
\hline & & & Mujer (1) \\
\hline & \multirow{5}{*}{ Rango de edad } & \multirow{5}{*}{$\begin{array}{l}\text { ¿Cuál es el intervalo que mejor } \\
\text { describe su edad? }\end{array}$} & 18 - 24 años (1) \\
\hline & & & 25 - 34 años (2) \\
\hline & & & 35 - 44 años (3) \\
\hline & & & 45 - 54 años (4) \\
\hline & & & 55 - 64 años (5) \\
\hline & \multirow{5}{*}{$\begin{array}{l}\text { Nivel de estudios com- } \\
\text { pletados }\end{array}$} & \multirow{5}{*}{$\begin{array}{l}\text { Nivel de estudios completado } \\
\text { actualmente }\end{array}$} & Sin estudios o primarios (1) \\
\hline & & & Estudios secundarios (2) \\
\hline & & & Estudios técnicos (3) \\
\hline & & & Estudios universitarios (4) \\
\hline & & & Estudios de posgrado (5) \\
\hline & \multirow{2}{*}{ Situación laboral } & \multirow{2}{*}{$\begin{array}{l}\text { ¿Cuál de las siguientes situaciones des- } \\
\text { cribe mejor su estatus laboral actual? }\end{array}$} & No trabaja (0) \\
\hline & & & Trabaja (1) \\
\hline & \multirow{3}{*}{$\begin{array}{l}\text { Rango de renta nacional } \\
\text { familiar anual }\end{array}$} & \multirow{3}{*}{$\begin{array}{l}\text { Tramo de renta anual de su hogar } \\
\text { incluyendo la suya y la de otros } \\
\text { posibles miembros de la familia }\end{array}$} & Tercio inferior nacional (1) \\
\hline & & & Tercio medio nacional (2) \\
\hline & & & Tercio superior nacional (3) \\
\hline & \multirow{2}{*}{$\begin{array}{l}\text { Creer poseer habilidades } \\
\text { para emprender }\end{array}$} & \multirow{2}{*}{$\begin{array}{l}\text { ¿Usted tiene los conocimientos, habi- } \\
\text { lidades y experiencia requerida para } \\
\text { poner en marcha un nuevo negocio? }\end{array}$} & No posee (0) \\
\hline & & & Sí posee (1) \\
\hline & \multirow{2}{*}{$\begin{array}{l}\text { Conocer personalmente a } \\
\text { un emprendedor }\end{array}$} & \multirow{2}{*}{$\begin{array}{l}\text { ¿Conoce personalmente a alguien } \\
\text { que haya puesto en marcha un nuevo } \\
\text { negocio en los últimos } 2 \text { años? }\end{array}$} & No conoce $(0)$ \\
\hline & & & Sí conoce (1) \\
\hline \multirow{3}{*}{ Regionales } & Localización & País de residencia & $\begin{array}{l}\text { Los siete (7) países de la } \\
\text { muestra }\end{array}$ \\
\hline & Inversión en I+D & \multicolumn{2}{|c|}{$\begin{array}{l}\text { Logaritmo natural de la inversión en I+D por habitante en Paridad de } \\
\text { Poder de Compra (PPC) al } 2010\end{array}$} \\
\hline & Oferta de capital riesgo & \multicolumn{2}{|c|}{$\begin{array}{l}\text { Logaritmo natural del número de ofertas de capital riesgo por trillón \$ } \\
\text { en PPC del PIB al } 2011\end{array}$} \\
\hline
\end{tabular}

Fuente: Elaboración propia.

Los valores medios de las variables utilizadas (cuadro 2) dan la pauta para construir el perfil del emprendedor tecnológico suramericano: se trata de un hombre, con edad promedio de 25 a 34 años, con un nivel educativo de hasta estudios secundarios, trabaja activamente y dispone de una renta familiar anual 
promedio correspondiente al tercio superior nacional. Adicionalmente, considera que tiene habilidades y conocimientos para emprender, y en su entorno familiar y social cuenta con referentes vinculados al emprendimiento.

Cuadro 2. Valores medios de las variables utilizadas (\% 2011)

\begin{tabular}{|c|c|c|c|c|c|}
\hline Variables & Categorías & $\begin{array}{c}\text { Muestra } \\
\text { total }\end{array}$ & \multirow{3}{*}{$\begin{array}{l}\text { Grupo: Em- } \\
\text { prendedor no } \\
\text { tecnológico }\end{array}$} & \multirow{3}{*}{$\begin{array}{c}\text { Grupo: } \\
\text { Emprendedor } \\
\text { tecnológico }\end{array}$} & \multirow{5}{*}{$\begin{array}{l}\text { Grupo: No } \\
\text { emprendedor }\end{array}$} \\
\hline \multirow{2}{*}{$\begin{array}{l}\text { Emprendimiento } \\
\text { (TEA11) }\end{array}$} & No emprendedor & 80.61 & & & \\
\hline & Emprendedor & 19.39 & & & \\
\hline \multirow{2}{*}{$\begin{array}{l}\text { Nivel tecnológico del em- } \\
\text { prendimiento } \\
\text { (TEA11TEC) }\end{array}$} & De nula o baja tecnología & 18.47 & 95.24 & & \\
\hline & De media o alta tecnología & 0.92 & & 4.76 & \\
\hline \multirow{2}{*}{ Género } & Hombre & 46.37 & 53.64 & 76.86 & 44.35 \\
\hline & Mujer & 53.63 & 46.36 & 23.14 & 55.65 \\
\hline \multirow{5}{*}{ Rango de edad } & 18-24 años & 19.97 & 15.87 & 22.00 & 20.95 \\
\hline & 25-34 años & 24.58 & 29.93 & 34.80 & 23.16 \\
\hline & 35-44 años & 22.79 & 27.09 & 24.40 & 21.73 \\
\hline & 45-54 años & 19.21 & 18.73 & 13.60 & 19.40 \\
\hline & 55-64 años & 13.44 & 8.38 & 5.20 & 14.77 \\
\hline \multirow{5}{*}{$\begin{array}{l}\text { Nivel de estudios comple- } \\
\text { tados }\end{array}$} & Sin estudios o primarios & 27.78 & 20.63 & 9.45 & 29.64 \\
\hline & Estudios secundarios & 42.81 & 41.70 & 36.61 & 43.13 \\
\hline & Estudios técnicos & 12.86 & 15.96 & 15.35 & 12.11 \\
\hline & Estudios universitarios & 13.13 & 17.10 & 32.68 & 11.99 \\
\hline & Estudios de posgrado & 3.43 & 4.62 & 5.91 & 3.13 \\
\hline \multirow{2}{*}{ Situación laboral } & No trabaja & 35.13 & 11.54 & 5.65 & 40.88 \\
\hline & Trabaja & 64.87 & 88.46 & 94.35 & 59.12 \\
\hline \multirow{3}{*}{$\begin{array}{l}\text { Rango de renta nacional } \\
\text { familiar anual }\end{array}$} & Tercio inferior nacional & 17.09 & 10.72 & 6.36 & 18.73 \\
\hline & Tercio medio nacional & 37.82 & 32.40 & 21.61 & 39.29 \\
\hline & Tercio superior nacional & 45.10 & 56.88 & 72.03 & 41.98 \\
\hline \multirow{2}{*}{$\begin{array}{l}\text { Creer poseer habilidades } \\
\text { para emprender }\end{array}$} & No posee & 40.14 & 14.10 & 14.74 & 46.45 \\
\hline & Sí posee & 59.86 & 85.90 & 85.26 & 53.55 \\
\hline \multirow{2}{*}{$\begin{array}{l}\text { Conocer personalmente a un } \\
\text { emprendedor }\end{array}$} & No conoce & 65.29 & 44.15 & 32.02 & 70.53 \\
\hline & Sí conoce & 34.71 & 55.85 & 67.98 & 29.47 \\
\hline \multicolumn{2}{|l|}{ Localización } & 4.31 & 4.21 & 4.11 & 4.34 \\
\hline \multicolumn{2}{|l|}{ Inversión en I+Di } & 53.10 & 51.55 & 51.90 & 53.47 \\
\hline \multicolumn{2}{|l|}{ Oferta de capital riesgo ii } & 9.95 & 8.87 & 9.30 & 10.20 \\
\hline
\end{tabular}

Notas: i No incluye a Perú y Venezuela, por no disponer de datos actualizados y por no contar con datos, respectivamente. ii No incluye a Chile y Venezuela por la inconsistencia de los datos.

Fuente: Elaboración propia.

\section{Metodología y resultados}

\section{Análisis econométrico multivariante}

Los modelos de estimación que se utilizan aquí responden a la naturaleza dicotómica de la variable dependiente, y se han llevado a cabo estimaciones con 
regresiones logísticas (modelos logit) en ambas submuestras. Por su parte, el análisis empírico se basa en la siguiente estimación:

$$
T E A_{i}=\alpha+\sum_{n} \beta_{n} X_{n, i}+\varepsilon_{i}
$$

donde $i$ se refiere al individuo; TEA es una variable dicotómica que hace referencia, en cada submuestra, a los emprendedores tecnológicos/no tecnológicos frente a los no emprendedores; $X n$ es el conjunto de variables independientes previamente definidas y $\varepsilon_{i}$ la perturbación aleatoria.

En el modelo 1 se analiza las variables individuales y se controla el efecto país incorporándolo en forma de variables dicotómicas regionales. En el modelo 2 se introducen las variables de control específicas regionales Gasto en $I+D$ y Oferta de capital riesgo en lugar de la variable país. En el modelo 3 se analiza las variables individuales y la variable regional Gasto en $I+D$, y es este el que se considera como más adecuado debido a que se ajusta de manera más realista al marco teórico de la investigación. Y en el modelo 4 se replica el uso de las variables del modelo anterior utilizando la submuestra de los "emprendedores no tecnológicos" para efectos comparativos y de medir diferencias.

Cuadro 3. Efectos marginales de las estimaciones sobre la probabilidad de ser emprendedor tecnológico

\begin{tabular}{|c|c|c|c|c|c|}
\hline & & \multicolumn{3}{|c|}{ Determinantes del emprendimiento tecnológico } & \multirow{3}{*}{$\begin{array}{c}\begin{array}{c}\text { Determinantes para } \\
\text { efectos comparativos }\end{array} \\
\text { Modelo } 4 \\
\begin{array}{c}\text { Emprendedor no } \\
\text { tecnológico }\end{array}\end{array}$} \\
\hline & & \multirow{2}{*}{$\begin{array}{c}\text { Modelo } 1 \\
\text { Emprendedor } \\
\text { tecnológico }\end{array}$} & \multirow{2}{*}{$\begin{array}{c}\text { Modelo } 2 \\
\text { Emprendedor } \\
\text { tecnológico }\end{array}$} & \multirow{2}{*}{$\begin{array}{c}\text { Modelo } 3 \\
\text { Emprendedor } \\
\text { tecnológico }\end{array}$} & \\
\hline Variables & Categorías & & & & \\
\hline $\begin{array}{l}\text { Género } \\
\text { (ref.: hombre) }\end{array}$ & Mujer & $\begin{array}{c}-0.0103^{\star \star \star} \\
(0.0016) \\
\end{array}$ & $\begin{array}{c}-0.0081^{\star \star \star} \\
(0.0017) \\
\end{array}$ & $\begin{array}{c}-0.0117^{\star \star \star} \\
(0.0017) \\
\end{array}$ & $\begin{array}{c}0.0032 \\
(0.0055) \\
\end{array}$ \\
\hline \multirow{4}{*}{$\begin{array}{l}\text { Rango de edad } \\
\text { (ref.: } 25 \text { - } 34 \text { años) }\end{array}$} & $\begin{array}{l}\text { Entre } 18 \text { - } 24 \\
\text { años }\end{array}$ & $\begin{array}{c}0.0041 \\
(0.0028) \\
\end{array}$ & $\begin{array}{l}0.0059+ \\
(0.0032) \\
\end{array}$ & $\begin{array}{l}0.0066^{\star} \\
(0.0032) \\
\end{array}$ & $\begin{array}{l}-0.0215^{\star \star} \\
(0.0082)\end{array}$ \\
\hline & $\begin{array}{l}\text { Entre } 35 \text { - } 44 \\
\text { años }\end{array}$ & $\begin{array}{l}-0.0020 \\
(0.0020) \\
\end{array}$ & $\begin{array}{c}-0.0060^{* * *} \\
(0.0019) \\
\end{array}$ & $\begin{array}{l}-0.0030 \\
(0.0021) \\
\end{array}$ & $\begin{array}{l}-0.0158^{*} \\
(0.0071) \\
\end{array}$ \\
\hline & $\begin{array}{l}\text { Entre } 45 \text { - } 54 \\
\text { años }\end{array}$ & $\begin{array}{c}-0.0060^{\star *} \\
(0.0019)\end{array}$ & $\begin{array}{l}-0.0030 \\
(0.0022)\end{array}$ & $\begin{array}{l}-0.0063^{\star \star} \\
(0.0021)\end{array}$ & $\begin{array}{l}-0.0361^{\star \star \star} \\
(0.0074)\end{array}$ \\
\hline & $\begin{array}{l}\text { Entre } 55 \text { - } 64 \\
\text { años }\end{array}$ & $\begin{array}{c}-0.0079^{* * *} \\
(0.0020)\end{array}$ & $\begin{array}{l}-0.0055^{*} \\
(0.0022)\end{array}$ & $\begin{array}{l}-0.0088^{* * *} \\
(0.0021)\end{array}$ & $\begin{array}{c}-0.0674^{\star \star \star} \\
(0.0084)\end{array}$ \\
\hline \multirow{4}{*}{$\begin{array}{l}\text { Nivel de estudios } \\
\text { completados } \\
\text { (ref.: estudios } \\
\text { secundarios) }\end{array}$} & $\begin{array}{l}\text { Sin estudios } \\
\text { o primarios }\end{array}$ & $\begin{array}{c}-0.0041+ \\
(0.0025)\end{array}$ & $\begin{array}{c}-0.0045+ \\
(0.0023)\end{array}$ & $\begin{array}{c}-0.0063^{\star *} \\
(0.0024)\end{array}$ & $\begin{array}{c}0.0007 \\
(0.0076)\end{array}$ \\
\hline & $\begin{array}{l}\text { Estudios } \\
\text { técnicos }\end{array}$ & $\begin{array}{c}0.0011 \\
(0.0027)\end{array}$ & $\begin{array}{c}0.0042 \\
(0.0034)\end{array}$ & $\begin{array}{c}0.0027 \\
(0.0031)\end{array}$ & $\begin{array}{c}0.0006 \\
(0.0080)\end{array}$ \\
\hline & $\begin{array}{l}\text { Estudios uni- } \\
\text { versitarios }\end{array}$ & $\begin{array}{l}0.0110^{* * *} \\
(0.0031)\end{array}$ & $\begin{array}{l}0.0110^{\star *} \\
(0.0041)\end{array}$ & $\begin{array}{l}0.0141^{* * *} \\
(0.0036)\end{array}$ & $\begin{array}{c}0.0049 \\
(0.0082)\end{array}$ \\
\hline & $\begin{array}{l}\text { Estudios de } \\
\text { posgrado }\end{array}$ & $\begin{array}{c}0.0031 \\
(0.0047)\end{array}$ & $\begin{array}{c}0.0048 \\
(0.0057)\end{array}$ & $\begin{array}{c}0.0052 \\
(0.0055)\end{array}$ & $\begin{array}{l}-0.0140 \\
(0.0129)\end{array}$ \\
\hline $\begin{array}{l}\text { Situación laboral } \\
\text { (ref.: no trabaja) }\end{array}$ & Sí trabaja & $\begin{array}{c}0.0111^{\star \star \star} \\
(0.0015)\end{array}$ & $\begin{array}{l}0.0109^{\star \star \star} \\
(0.0014)\end{array}$ & $\begin{array}{c}0.0120^{\star * *} \\
(0.0016)\end{array}$ & $\begin{array}{c}0.1506^{\star * *} \\
(0.0057)\end{array}$ \\
\hline
\end{tabular}

10 G. Zapata, S. Fernández López, I. Neira Gómez | El emprendimiento tecnológico en Suramérica: una aproximación a sus determinantes individualesa | Perfiles Latinoamericanos, 26(52) | FLACso México DOI: $10.18504 / \mathrm{pl} 2652-003-2018$ 
Cuadro 3. Continuación

\begin{tabular}{|c|c|c|c|c|c|}
\hline & & \multicolumn{3}{|c|}{ Determinantes del emprendimiento tecnológico } & \multirow{3}{*}{$\begin{array}{c}\begin{array}{c}\text { Determinantes para } \\
\text { efectos comparativos }\end{array} \\
\text { Modelo } 4 \\
\begin{array}{c}\text { Emprendedor no } \\
\text { tecnológico }\end{array}\end{array}$} \\
\hline & & \multirow{2}{*}{$\begin{array}{c}\text { Modelo } 1 \\
\text { Emprendedor } \\
\text { tecnológico }\end{array}$} & \multirow{2}{*}{$\begin{array}{c}\text { Modelo } 2 \\
\text { Emprendedor } \\
\text { tecnológico }\end{array}$} & \multirow{2}{*}{$\begin{array}{c}\text { Modelo } 3 \\
\text { Emprendedor } \\
\text { tecnológico }\end{array}$} & \\
\hline Variables & Categorías & & & & \\
\hline \multirow{2}{*}{$\begin{array}{l}\text { Rango de renta } \\
\text { nacional familiar } \\
\text { anual } \\
\text { (ref.: tercio medio } \\
\text { nacional) }\end{array}$} & $\begin{array}{l}\text { Tercio infe- } \\
\text { rior nacional }\end{array}$ & $\begin{array}{l}-0.0004 \\
(0.0038)\end{array}$ & $\begin{array}{c}0.0017 \\
(0.0045)\end{array}$ & $\begin{array}{c}0.0033 \\
(0.0050)\end{array}$ & $\begin{array}{l}-0.0053 \\
(0.0094)\end{array}$ \\
\hline & $\begin{array}{l}\text { Tercio } \\
\text { superior } \\
\text { nacional }\end{array}$ & $\begin{array}{l}0.0054^{* *} \\
(0.0019)\end{array}$ & $\begin{array}{l}0.0036+ \\
(0.0020)\end{array}$ & $\begin{array}{l}0.0060^{\star *} \\
(0.0020)\end{array}$ & $\begin{array}{l}0.0383^{\star \star *} \\
(0.0063)\end{array}$ \\
\hline $\begin{array}{l}\text { Creer poseer } \\
\text { habilidades para } \\
\text { emprender } \\
\text { (ref.: no posee) }\end{array}$ & Sí posee & $\begin{array}{c}0.0101^{\star \star \star} \\
(0.0015)\end{array}$ & $\begin{array}{l}0.0085^{\star * *} \\
(0.0017)\end{array}$ & $\begin{array}{c}0.0117^{\star \star \star} \\
(0.0016)\end{array}$ & $\begin{array}{l}0.1687^{\star \star \star} \\
(0.0053)\end{array}$ \\
\hline $\begin{array}{l}\text { Conocer perso- } \\
\text { nalmente a un } \\
\text { emprendedor } \\
\text { (ref.: no conoce) }\end{array}$ & Sí conoce & $\begin{array}{c}0.0131^{* * *} \\
(0.0019)\end{array}$ & $\begin{array}{l}0.0085^{\star \star *} \\
(0.0020)\end{array}$ & $\begin{array}{l}0.0131^{\star \star \star} \\
(0.0020)\end{array}$ & $\begin{array}{l}0.1110^{\star \star \star} \\
(0.0060)\end{array}$ \\
\hline \multirow{6}{*}{$\begin{array}{l}\text { País } \\
\text { (ref.: Venezuela) }\end{array}$} & Perú & $\begin{array}{c}0.0083 \\
(0.0108)\end{array}$ & & & \\
\hline & Argentina & $\begin{array}{c}0.0187 \\
(0.0152)\end{array}$ & & & \\
\hline & Brasil & $\begin{array}{l}-0.0061 \\
(0.0047)\end{array}$ & & & \\
\hline & Chile & $\begin{array}{l}0.0199+ \\
(0.0113)\end{array}$ & & & \\
\hline & Colombia & $\begin{array}{c}0.0096 \\
(0.0083)\end{array}$ & & & \\
\hline & Uruguay & $\begin{array}{c}0.0124 \\
(0.0127)\end{array}$ & & & \\
\hline \multicolumn{2}{|l|}{ Inversión en I+D } & & $\begin{array}{l}-0.0026 \\
(0.0018)\end{array}$ & $\begin{array}{c}0.0014 \\
(0.0012)\end{array}$ & $\begin{array}{l}-0.0047 \\
(0.0035)\end{array}$ \\
\hline \multicolumn{2}{|c|}{ Oferta de capital riesgo } & & $\begin{array}{c}0.0021 \\
(0.0018)\end{array}$ & & \\
\hline \multicolumn{2}{|c|}{ Número de observaciones } & 17677 & 11325 & 15665 & 19417 \\
\hline \multicolumn{2}{|c|}{ test de verosimilitud chi-2 } & 429.15 & 218.26 & 396.57 & 2829.98 \\
\hline \multicolumn{2}{|l|}{ Grados de libertad } & 20 & 16 & 15 & 15 \\
\hline \multicolumn{2}{|l|}{$-2 L L$} & -969.05783 & -496.18984 & -889.95876 & -8401.1228 \\
\hline \multicolumn{2}{|l|}{$p$-valor } & 0 & 0 & 0 & 0 \\
\hline \multicolumn{2}{|l|}{ Pseudo-R2 } & 0.1813 & 0.1803 & 0.1822 & 0.1441 \\
\hline
\end{tabular}

Nota: Errores estándar entre paréntesis; $+p<0.10 ;{ }^{*} p<0.05 ;{ }^{* *} p<0.01 ;{ }^{* * *} p<0.001$

Fuente: Elaboración propia.

\section{Descripción de resultados}

En los resultados se puede observar que en Suramérica hay un efecto negativo y altamente significativo sobre las probabilidades de ser emprendedor tecnológico en iniciativas empresariales generadas por mujeres. Influencia que no se replica para el emprendimiento convencional (no tecnológico); al parecer 
en este caso ser hombre o mujer es indiferente pues no se evidencia efecto significativo alguno en los resultados.

La edad de los emprendedores tecnológicos con referencia al segundo intervalo (25-34 años) influye positiva y significativamente sobre la probabilidad de emprender cuando ellos son menores (18-24 años). Pero cuando se superan los 45 años de edad la influencia es negativa. En contraste, en el emprendimiento no tecnológico aparecen dos diferencias: ser menor (18-24 años) resulta negativo y significativo, de la misma manera que el rango inmediatamente superior al de referencia.

El efecto del nivel de estudios alcanzados sobre la probabilidad de iniciar un emprendimiento tecnológico, tomando como referencia los estudios secundarios, indica que no poseer estudios o solo estudios primarios influye negativa y significativamente. Mientras que contar con nivel universitario favorece las probabilidades del emprendimiento tecnológico. Por su parte, en el emprendimiento convencional, sea cual sea el nivel de estudios alcanzado, no hay efecto sobre la probabilidades favorables de emprender.

Respecto al factor experiencia laboral, que toma como proxy la variable $s i$ tuación laboral, los resultados muestran que estar en situación de empleo activo a tiempo parcial o completo tiene una influencia positiva y altamente significativa en las probabilidades de emprender negocios de base tecnológica, y que en cuanto a los no tecnológicos, el efecto es el mismo en idénticos términos (signo y significatividad), aunque con un mayor impacto entre estos últimos, los no tecnológicos.

La renta familiar anual disponible, con referencia al tercio medio nacional de los ingresos, indica que cuando el individuo percibe ingresos del rango del tercio superior nacional surge una influencia positiva y altamente significativa sobre la probabilidad de emprender en el ámbito tecnológico. Respecto a los emprendedores no tecnológicos, el efecto de la renta es similar, esto es, indiferente cuando es inferior y significativamente positiva cuando es superior, e incluso esta última parece acrecentarse en este segundo caso.

En cuanto a aquellos que creen poseer habilidades especificas para emprender, se muestra un efecto positivo y significativo sobre las probabilidades de iniciar un negocio de base tecnológica, aunque el impacto es mayor impacto en las iniciativas empresariales no tecnológicas.

Contar con alguna proximidad o conocer personalmente a individuos que han estado o están involucrados en algún tipo de emprendimiento denota, según los resultados, una influencia positiva y significativa sobre las probabilidades favorables de que el individuo inicie emprendimientos de base tecnológica, influencia que comparten los emprendedores no tecnológicos, entre los que incluso es mayor el impacto. 
Acerca de la variable de control regional inversión en $I+D$ no se halló efecto significativo alguno ni para el emprendimiento tecnológico ni para el convencional.

Cabe mencionar que, a pesar de la preferencia por el modelo 3, en el modelo 1 se trató de identificar algún efecto país a manera de aproximación a algunas diferencias entre los países sobre las probabilidades de crear una NEBT. Tomando como referencia a Venezuela, país con el más bajo nivel de emprendimiento $(1.32 \%)$, no se hallaron efectos significativos, salvo en Chile con un efecto positivo, aunque con un margen de error del 10\%. Sin embargo, aunque no se recoge en el cuadro 3 , sí se hallaron diferencias significativas positivas para el emprendedor no tecnológico en Argentina, Chile y Colombia.

Del mismo modo, en el modelo 2 se mide el efecto de la variable de control regional oferta de capital riesgo sobre las probabilidades favorables de creación de NEBT, pero tampoco hubo trazas de efectos significativos, aunque sí, y de forma positiva, para los emprendedores convencionales.

\section{Análisis y discusión de resultados}

El género demuestra empíricamente que es un factor determinante entre los individuos suramericanos cuando estos deciden un emprendimiento de base tecnológica. Así, la presencia femenina se encuentra asociada a un efecto negativo y altamente significativo sobre la probabilidad de iniciar una NEBT, lo que confirma en parte las barreras señaladas en Rodríguez et al. (2013), Mayer (2008) y Zhao et al. (2005): la falta de modelos femeninos a raíz de la alta percepción de un entorno fuertemente competitivo y por eso de mayor dificultad y menos apropiado para emprender, además de la difícil conciliación de la vida laboral con la familiar. Frente a este resultado, el género parece no influir significativamente en el emprendimiento no tecnológico.

Pero la edad, en relación con el emprendimiento tecnológico en Suramérica, contrario a lo esperado, se asocia positivamente con tener edades menores (1834 ańos), lo que refuta a Westhead \& Storey (1994), Harvey (1994), Donckels (1989), Autio et al. (1989), Ortín et al. (2008) y Zapata et al. (2014). Storey \& Tether (1998) ya habían planteado la poca probabilidad de encontrar emprendedores muy jóvenes, es decir, con edades menores a los 25 años. Los resultados de esta investigación se explican en cierto aspecto por la más intensa vinculación de los jóvenes con el manejo de las nuevas tecnologías. Su única diferencia respecto de los emprendedores no tecnológicos es que las edades de estos últimos son del rango 25-34 años, con lo que tienen menor margen de tiempo para emprender.

El efecto del nivel de estudios alcanzados en emprendimientos tecnológicos coincide parcialmente con la literatura. Storey \& Tether (1998), Westhead \& 
Storey (1994) y Ortín et al. (2008) han vinculado la creación de NEBT con niveles educativos de estudios superiores a los secundarios (técnicos, universitarios y posgrado). Nuestra evidencia empírica demuestra que, en Suramérica, de esa afirmación solo el tener estudios universitarios muestra una influencia positiva y significativa. Estos resultados tal vez se deben a que en la región suramericana, quien tiene estudios técnicos es más probable que encuentre un empleo como asalariado en industrias que están desarrollándose. Asimismo, aquellos con posgrado están muy vinculados a la vida académica o tienen altas expectativas de posicionamiento en la institución donde trabajan. Sin embargo, solo los que cuentan con estudios universitarios tendrían más latente la alternativa de emprender un negocio como opción profesional.

La variable situación laboral, incorporada como proxy de la experiencia, demostró una influencia positiva en la puesta en marcha de un negocio en sectores de media y alta tecnología. Esto corroboraría los argumentos de quienes sostienen que el aprendizaje que el emprendedor comporta de situaciones laborales previas tiene un efecto positivo (Colombo \& Grilli, 2005). Pero, en sentido opuesto al esperado, el efecto positivo de la experiencia laboral es mayor en el emprendimiento no tecnológico. Es posible que esto se deba a que es más fácil trasladar dicha experiencia en el emprendimiento de negocios convencionales que en iniciativas tecnológicas.

Ahora bien, cuando es alta la renta familiar anual del individuo, es decir, cuando pertenece al tercio superior nacional, hay un efecto positivo en la probabilidad de que él emprenda una NEBT. Esto confirma los estudios previos, en los cuales se sostiene que las "restricciones financieras" actúan como barrera para el comienzo de negocios tecnológicos, pues se debe recurrir a la riqueza personal (Colombo \& Grilli, 2005). Esta idea parece confirmarse si se observa que la renta familiar ha mostrado ese mismo efecto positivo y significativo en el emprendimiento convencional. No obstante, su magnitud no es la esperada, dado que es mayor en el emprendimiento convencional. Este hallazgo contraviene lo postulado por Ortín et al. (2008) para las NEBT; estos autores han señalado que debido a que al emprendimiento se le asocian el riesgo y la incertidumbre en los resultados, el acceso al crédito es restringido.

La creencia de los emprendedores de poseer habilidades (y conocimientos) para comenzar una iniciativa empresarial tiene efecto positivo en la creación de empresas de base tecnológica, lo que concuerda con lo esperado. Creer que estas habilidades combinadas con los conocimientos pueden llevar a crear valor, anima a los individuos a iniciar NEBT (Shane \& Venkataraman, 2000; Álvarez \& Barney, 2002). Este impacto positivo se repite, pero más intenso, para el emprendimiento no tecnológico. 
Finalmente, conocer personalmente a un emprendedor ha mostrado un efecto positivo para los emprendimientos de negocios de base tecnológica, lo que confirma su influencia en las actitudes, hábitos y habilidades para los emprendedores, sobre todo en cuestiones de gestión (Gibb, 1997). El influjo de personas muy próximas - del entorno familiar o de las redes sociales — es determinante si se repara en que en estos espacios esas figuras cumplen un rol de modelo para los potenciales emprendedores (Roberts, 1991). Es un efecto positivo que cobra aún más importancia entre los emprendedores no tecnológicos.

Por su parte, los factores regionales, las variables de control gasto en $\mathrm{I}+\mathrm{D}$ y la oferta de capital riesgo no han mostrado efecto significativo alguno. No es posible confirmar entonces los postulados que afirman su influencia sobre el emprendimiento tecnológico. No obstante, estos resultados adversos podrían responder más a cuestiones técnicas de medición de datos y disposición de estos que a la no corrobación de resultados empíricos de estudios previos.

\section{Conclusiones}

En el presente artículo, con datos del proyecto Gem para Suramérica (Argentina, Brasil, Chile, Colombia, Perú, Uruguay y Venezuela) para 2011, se han analizado los factores individuales que determinan la intención del individuo suramericano de emprender una NEBT, y en qué medida esto difiere frente a los emprendedores convencionales. De esta manera ampliamos la comprensión del emprendimiento tecnológico con el fin de contribuir a la construcción de condiciones favorables para su desarrollo que permitan encauzar a la sociedad suramericana en relación con las sociedades del conocimiento. Es una forma de buscar que la base de nuevos negocios esté vinculada a conocimientos tecnocientíficos que den soporte al aceleramiento del proceso transitorio de la región suramericana hacia una economía basada en la innovación.

En cuanto al objetivo propuesto, los factores individuales vinculados positivamente a las probabilidades de iniciar una NEBT son el tener una edad en el rango 18-24 años, poseer estudios universitarios, mantener una situación laboral activa (de tiempo parcial o completo), disponer de una renta familiar anual perteneciente al tercio superior nacional, contar con la autopercepción de poseer habilidades y conocimientos para emprender, y conocer a personas que en algún momento han sido emprendedoras. Por el contrario, los factores que afectan negativamente en las probabilidades de emprender en sectores tecnológicos serían: ser mujer, tener una edad superior a los 45 años y carecer de estudios superiores, o solo haber cursado los primarios. Se ha encontrado además que el emprendedor tecnológico muestra rasgos que lo diferencian de su 
par no tecnológico. Así, solo para los emprendedores no tecnológicos: 1) el hecho de estar fuera del rango de edad de 25 a 34 afecta negativamente sus probabilidades de emprender una NEBT, mientras que 2) ello no se afecta por el nivel de estudios del emprendedor. De igual modo, estar trabajando, poseer habilidades (y conocimientos) para emprender y contar con antecedentes familiares de emprendimiento influye positivamente — como sucede en el emprendimiento tecnológico-, pero con un efecto superior.

Este trabajo no está exento de limitaciones que pueden significar líneas de investigación futuras. Así, aunque el enfoque aquí ha tomado como objeto de estudio a los individuos, se debería contextualizar más a fondo el territorio en el que surgen los emprendimientos tecnológicos, pues ese aspecto define las características de los ecosistemas de innovación que contribuyen a la creación de NEBT. En investigaciones futuras se podría ahondar en el factor contextual/ regional, para incorporar incluso a aquellos de carácter intangible o cultural, pero estrechamente vinculados al emprendimiento tecnológico. Futuras investigaciones, asimismo, podrían beneficiarse del uso de metodologías más apropiadas, pero que están en desarrollo, por ejemplo el análisis multinivel, para capturar los factores contextuales.

\section{Referencias}

Aceytuno Pérez, M. T. \& Paz Báńez, M. A. de (2008). La creación de spin-off universitarias. El caso de la Universidad de Huelva. Economía Industrial, (368), 97-111.

Aldrich, H. (2012). The emergence of entrepreneurship as an academic field: A personal essay on institutional entrepreneurship. Research Policy, 41(7), 1240-1248.

Aldrich, H. \& Fiol, M. (1994). Fools rush in? The institutional context of industry creation. The Academy of Management Review, 19(4), 645-670.

Aldrich, H. \& Zimmer, C. (1986). Entrepreneurship through Social Networks. En The Art and Science of Entrepreneurship (pp. 3-23). Cambridge: Ballinger Publishing Co.

Almus, M. \& Nerlinger, E. (1999). Growth of new technology-based firms: Which factors matter? Small Business Economics, 13(2), 141-154.

Alonso, M., Galve, C. \& Hernandez, A. (2006). Creación de Empresas y autoempleo en Europa: periodo 1997-2002. En XVI Jornadas Luso-Espanholas Gestao Cientifica. Inovação e Empreendedorismo para o Século XXI (pp. 354-364). Évora: Universidad de Évora. 
Alvarez, C. \& Urbano, D. (2011). Environmental factors and entrepreneurial activity in Latin America. Academia Revista Latinoamericana de Administración, 48, 31-45.

Alvarez, S. \& Barney, J. (2002). Resource-based theory and the entrepreneurial firm. En Hitt, M., Ireland, R., Camp, S. \& Sexton, D. (Eds.). Strategic Entrepreneurship (pp. 89-105). Oxford: Blackwell Publishers.

Aspelund, A., Berg-Utby, T. \& Skjevdal, R. (2005). Initial resources' influence on new venture survival: A longitudinal study of new technology-based firms. Technovation, 25(11), 1337-1347.

Autio, E. (1997). Atomistic and systemic approaches to research on new, technology-based firms: A literature study. Small Business Economics, 9(3), 195-209.

Autio, E., Kanerva, R., Kaila, M. \& Kauranen, I. (1989). Uudet teknologiayritykset (New, Technology-Based Firms. Helsinki: sitra Publication.

Bandura, A. (1977). Social Learning Theory. Englewood Cliffs, N. J.: Prentice Hall.

Bower, D. (2003). Business model fashion and the academic spinout firm. R\&D Management, 33(2), 97-106.

Cepal. (2014). Estudio Económico de América Latina y el Caribe. Recuperado de http://www .cepal.org/publicaciones/xml/2/53392/EstudioEconomico2014.pdf

Clarysse, B. \& Moray, N. (2004). A Process Study of Entrepreneurial Team Formation: The Case of a Research-based Spin-off. Journal of Business Venturing, 19(1), 55-79.

Colombo, M. \& Grilli, L. (2005). Founders' human capital and the growth of new technology based firms: a competence-based view. Research Policy, 34(6), 795-816.

Cooper, A. C. (1971). The founding of technologically-Based Firms. Milwaukee: The Center for Venture Management.

Costa, F. (2006). O capital de risco, a incubacao de empresas e o emprendedorismo: os criterios de seleccao de projectos. XVI Jornadas Luso-Espanholas Gestao Cientifica. Inovação e Empreendedorismo para o Século XXI (pp. 313-323). Évora: Universidad de Évora.

Cunha, D., Silva, S. \& Teixeira, A. (2013). Are Academic Spin-Offs necessarily New TechnologyBased firms? (Working Paper 482), Porto: Faculdade de Economia do Porto, Universidade do Porto. 
Davidsson, P. \& Honig, B. (2003). The role of social and human capital among nascent entrepreneurs. Journal of Business Venturing, 18(3), 301-331.

Donckels, R. (1989). Tech versus Common Starters: comparison by means of 32 case studies. Bruselas: Small Business Research Institute.

Fagenson, E. \& Jackson, J. (1993). The status of women managers in the United States. International Studies of Management \& Organization, 23(2), 93-112.

Ferreira, J., Raposo, M., Ferreira, F., Jalali, M., Fernandes, C. \& Marques, C. (2014). What do we Know About Technology Entrepreneurship Research? 2014 INBAM Conference Annual Meeting, Barcelona.

Gassler, H. (1998). The regional variation of new technology based firms in Austria. ERSA Conference Papers 38th. Viena: ERSA.

Gibb, A. (1997). Small Firms Training and Competitiveness. Building Upon the Small Business as a Learning Organisation. International Small Business Journal, 15(3), 12-29.

Giovannetti, G., Ricchiuti, G. \& Velucchi, M. (2011). Size, innovation and internationalization: A survival analysis of Italian firms. Applied Economics, 43(12), 1511-1520.

Goldfarb, B. \& Henrekson, M. (2003). Bottom-up versus top-down policies towards the commercialization of university intellectual property. Research Policy, 32(4), 639-658.

Goldin, C. (2006). The quiet revolution that transformed women's employment, education, and family. (Working Paper no. 11953). Cambridge, M. A.: National Bureau of Economic Research.

Gompers, P., Lerner, J. \& Scharfstein, D. (2005). Enterpreneurial Spawning: Public Corporations and the genesis of New Ventures, 1986 to 1999. The Journal of Finance, 60(2), 577-614.

Greene, P. (2000). Self-employment as an economic behavior: an analysis of self-employed women's human and social capital. National Journal of Sociology, 12(1), 1-55.

Harvey, K. (1994). From Handicap to Nice Little Earner: A Study of Academic Spin-Off Enterprise. (Paper). Manchester Business School Conference.

Hülsbeck, M. \& Kitzinger, E. (2011). The impact of regional industries and universities on (high) technology entrepreneurship. (UO Working Paper Series no. 03-11). Augsburg: University of Augsburg.

18 - G. Zapata, S. Fernández López, I. Neira Gómez | El emprendimiento tecnológico en Suramérica: una aproximación a sus determinantes individualesa | Perfiles Latinoamericanos, 26(52) | FLacso México DOI: $10.18504 / \mathrm{pl} 2652-003-2018$ 
Jack, S. \& Anderson, A. (2002). The effects of embeddedness on the entrepreneurial process. Journal of Business Venturing, 17(5), 467-487.

Lechner, C., Dowling, M. \& Welpe, I. (2006). Firm networks and firm development: The role of the relational mix. Journal of Business Venturing, 21(4), 514-540.

Lederman, D., Messina, J., Pienknagura, S. \& Rigolini, J. (2014). El emprendimiento en América Latina: muchas empresas y poca innovación. Washington, D. C.: Banco Mundial.

Mayer, H. (2008). Segmentation and Segregation Patterns of Women-Owned High-Tech Firms in Four Metropolitan Regions in the United States. Regional Studies, 42(10), 1357-1383.

Murray, F. \& Graham, L. (2007). Buying science and selling science: gender differences in the market for commercial Science. Industrial and Corporate Change, 16(4), 657-689.

Neergaard, H., Nielsen, K. \& Kjeldsen, J. (2006). State of the art of women's entrepreneurship, access to financing and financing strategies in Denmark. En Brush, C., Carter, N., Gatewood, E., Greene P. \& Hart, M. (Eds.). Growth-oriented Women Entrepreneurs and Their Businesses: A Global Research Perspective (pp. 88-111). Cheltenham, Reino Unido: Edward Elgar,

O'shea, R., Chugh, H. \& Allen, T. (2008). Determinants and consequences of university spinoff activity: a conceptual framework. The Journal of Technology Transfer, 33(6), 653-666.

Oakey, R. (1995). High technology new firms: variable barriers to growth. Londres: Paul Chapman Publishing.

OCDE. (2009). Innovation in firms: A microeconomic perspective. Recuperado de http://browse .oecdbookshop.org/oecd/pdfs/browseit/9209011E.PDF.

oecd/eclac. (2012). Perspectivas económicas de América Latina 2013: Politicas de pymes para el cambio estructural. Recuperado de http://www.cepal.org/publicaciones/xml/4/48374/ leo_2013.pdf

Ortín, P., Salas, V., Trujillo, M. \& Vendrell, F. (2008). La creación de spin-off universitarios en España: características, determinantes y resultados. Economía Industrial, (368), 79-95.

Petti, C. \& Zhang, S. (2011). Factors influencing technological entrepreneurship capabilities: Towards an integrated research framework for Chinese enterprises. Journal of Technology Management in China, 6(1), 7-25.

Roberts, B. (1991). Entrepreneurs in High Technology: Lessons from MIT and Beyon. Nueva York: Oxford University Press. 
Rodríguez, M., Fernández, S., Rodeiro, D. \& Vivel, M. (2013). Emprendimiento tecnológico ¿Una cuestión de género también en la universidad? XXIII Jornadas Hispanolusas. Bases para Emprender y Gestionar con Racionalidad y Transparencia (pp. 55-65). Málaga: Universidad de Málaga.

Rueda, I., Fernández-Laviada, A. \& Herrero, A. (2014). Entrepreneurial intention: Perceived advantages and disadvantages. Academia Revista Latinoamericana de Administración, 27(2), 284-315.

Ruiz, J., Camelo, M. \& Coduras, A. (2012). Mujer y desafío emprendedor en España. Características y determinantes. Economía Industrial, (383), 13-22.

Shane, S. \& Venkataraman, S. (2000). The promise of entrepreneurship as a field of research. Academy of Management Review, 25(1), 217-226.

Storey, D. \& Tether, B. (1998). New technology-based firms in the European Union: An introduction. Research Policy, 26(9), 933-946.

Vaquero, A., Fernández, S., Vivel, M., López, A., Porto, N. \& Filgueira, A. (2011). El papel de la mujer en el campo científico, tecnológico y de transferencia del conocimiento en Galicia. Ourense.

Venkataraman, S. (2004). Regional transformation through technological entrepreneurship. Journal of Business venturing, 9(1), 153-167.

Westhead, P. \& Storey, D. (1994). An Assessment of Firms Located On and Off Science Parks in the United Kingdom. Londrers: HM Stationery Office.

Xue, J. \& Klein, P. (2010). Regional determinants of technology entrepreneurship. International Journal of Entrepreneurial Venturing, 1(3), 291-308.

Zapata, G., Fernández, S., Vivel, M., Neira, I. \& Rodeiro, D. (2014a). El emprendimiento de base tecnológica; Características diferenciales. En Vence, X. \& Rodeiro, D. (Coords.). Innovación y emprendimiento con base en las ciencias (pp. 3-22). Santiago de Compostela: Universidad de Santiago de Compostela.

Zapata, G., Fernández, S., Neira, I. \& Nogueira, M. (2014b). Factores individuales determinantes del emprendimiento tecnológico: un análisis del caso español. XXIV Jornadas Luso-Espanholas. Gestão Científica. O Contributo da Gestão para a Sustentabilidade das Organizaçóes e da Sociedade. Leiria, Portugal: Instituto Politécnico de Leiria.

Zhao, H., Scott, E. \& Hills, G. (2005). The Mediating Role of Self-Efficacy in the Development of Entrepreneurial Intentions. Journal of Applied Psychology, 90(6), 1265-1272.

20 G. Zapata, S. Fernández López, I. Neira Gómez | El emprendimiento tecnológico en Suramérica: una aproximación a sus determinantes individualesa | Perfiles Latinoamericanos, 26(52) | FLacso México DOI: $10.18504 / \mathrm{pl} 2652-003-2018$ 